COMMUNICATIONS ARISING Earth science

\section{Mantle deformation or processing artefact?}

M

easurements of shear-wave splitting represent an important tool for determining seismic anisotropy and for quantifying deformation processes in the Earth. Wookey et al. ${ }^{1}$ claim to have observed significant seismic anisotropy in the mid-mantle between the Tonga-Kermadec subduction zone and Australia. We argue that their results are likely to be methodological artefacts and that the available data can be explained by moderate anisotropy in the upper mantle close to the seismograph stations. The lack of evidence for anisotropy in the mid-mantle nullifies any related geodynamic inferences.

Elastic anisotropy results in different propagation velocities of shear $(S)$ waves with different polarizations. Wookey et al. ${ }^{1}$ report 2-7 seconds of splitting for 15 of 35 S-waves from deep earthquakes, recorded in Australia at epicentral distances of $24-59^{\circ}$. Their observation that $\mathrm{SH}$ (motion perpendicular to the wave-propagation plane) generally leads SV (motion within the wave-propagation plane) is interpreted as transverse isotropy (or radial anisotropy with a vertical slow axis) along subhorizontal parts of the wavepaths. This type of anisotropy is well known at the bottom of the lower mantle ${ }^{2}$, but not in the mid-mantle.

To measure S-wave splitting, Wookey et al. ${ }^{1}$ adopted a method that is normally used to study mantle anisotropy using SKS waveforms, which travel through the Earth's core and become pure SV waves upon reentering the mantle. The presence of mantle anisotropy beneath a station causes SKS to split into a fast and slow quasi-S-wave, the amount of splitting allowing quantification of the anisotropy. This technique requires S-waves that are steeply incident at the receiver, resulting in almost pure SV motion

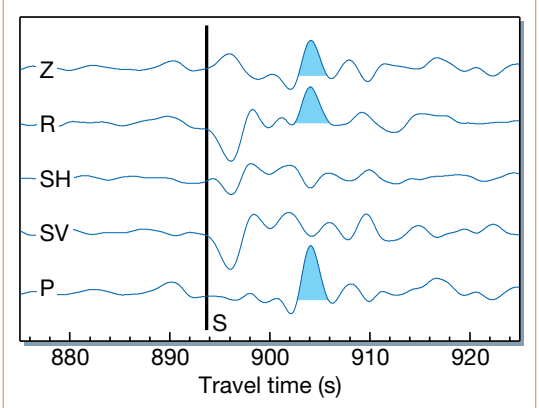

Figure 1 Example of a recording used by Wookey et al. ${ }^{1}$ (obtained on 13 April 1995 at station NWAO), who analysed traces labelled $R$ and $\mathrm{SH}$ and obtained a splitting time of $4.35 \mathrm{~s}$. A shear-coupled P-wave (shaded) severely contaminates the radial (R) component. SV and SH have very similar waveforms and do not show any difference in travel time. All traces are plotted at the same amplitude scale. on the radial component and negligible interactions with near-receiver structure.

The main obstacle to the approach of Wookey et al. ${ }^{1}$ is the presence of largeamplitude shear-coupled compressional (P) waves thoughout their data set. At epicentral distances of less than $60^{\circ}$, these reverberation phases represent a large percentage of the energy recorded on the radial component ${ }^{3,4}$ (Fig. 1). Wookey et al. ${ }^{1}$ mistakenly invert this mixture of direct SV and shear-coupled P-waves as pure SV motion, rendering their splitting measurements meaningless.

Through comparison of $\mathrm{SH}$ and $\mathrm{SV}$ waveforms, which is a standard technique to investigate transversely isotropic media, we test the proposal put forward by Wookey et al. ${ }^{1}$. To avoid contamination of the SV signal by $\mathrm{P}$ phases, we decompose the $\mathrm{P}$ and SV wavefields through further rotation of radial and vertical components ${ }^{5}$ (Fig. 1). Examining all of the available data for the stations, we found 128 recordings with qualities that are comparable to those of the 35 used by Wookey et al.

Our results are very different from those of Wookey et al. Splitting times are consistently small and strongly station-dependent, ranging from almost zero at station CTAO to $1.3 \mathrm{~s}$ at WRAB and TAU. At stations NWAO and CAN, averages amount to 0.4 and $0.7 \mathrm{~s}$, respectively. Wherever splitting is detectable, the fast axis coincides with SH. Owing to their shallow incidence in the upper mantle, S-waves, as opposed to SKS, are sensitive to radial anisotropy. The relatively small splitting times can therefore be explained entirely by a radially anisotropic uppermost mantle ${ }^{6}$ and do not require anisotropy at mid-mantle level.

\section{Joachim Saul ${ }^{\star}$, Lev Vinnik ${ }^{\star} \dagger$}

${ }^{\star}$ GeoForschungsZentrum Potsdam, Telegrafenberg, 14473 Potsdam, Germany

e-mail: saul@gfz-potsdam.de

$\dagger$ Institute of Earth Physics, Moscow 123995, Russia

$$
\begin{aligned}
& \text { 1. Wookey, J., Kendall, J.-M. \& Barruol, G. Nature 415, } \\
& 777-780 \text { (2002). } \\
& \text { 2. Vinnik, L., Breger, L. \& Romanowicz, B. Nature 393, } \\
& \text { 564-567 (1998). } \\
& \text { 3. Båth, M. \& Stefansson, R. Ann. Geofis. 19, 119-130 (1966). } \\
& \text { 4. Zandt, G. \& Randall, G. E. Geophys. Res. Lett. 12, } \\
& \text { 565-568 (1985). } \\
& \text { 5. Bostock, M. G. J. Geophys. Res. 103, 21183-21200 (1998). } \\
& \text { 6. Gaherty, J. B. \& Jordan, T. H. Science 268, 1468-1471 (1995). }
\end{aligned}
$$

Wookey et al. reply — Saul and Vinnik's comment raises an interesting point concerning the influence of shear-coupled P-waves (such as SPmP) on shear-wavesplitting analysis. We used a method ${ }^{1,2}$ that applies a time window to the data, so Pwave energy on the horizontal components outside this window will have no effect on the analysis. As all but one of our events were recorded at stations located on thick (40-50 km) continental crust ${ }^{3}$, the Moho multiple arrivals to which we refer will generally be separated ( $>8 \mathrm{~s}$ for SPmP) from the main $\mathrm{S}$-wave arrival. Error can occur when P-wave energy falls within the analysis window.

We have re-analysed our data, applying a wavefield-decomposition technique ${ }^{4}$ before the shear-wave splitting analysis. The range of results changed from $0.6-7.1 \mathrm{~s}$ to $0.6-6.2 \mathrm{~s}$, with ten results showing splitting greater than $1.5 \mathrm{~s}$. This is more splitting than can be explained solely by VTI (transverse isotropy with a vertical axis of symmetry) in the upper mantle and still supports our conclusion of mid-mantle anisotropy.

The mistake that Saul and Vinnik have made is to assume a VTI medium and simply to look for splitting between the radial and transverse components. Although our results show a near-VTI symmetry on average $\left(\varphi_{\mathrm{AVE}}=95.3^{\circ}\right)$, there is plenty of scatter in the data. The lack of apparent splitting on the components of the seismogram (Fig. 1) is meaningless - the arrivals on the radial and transverse components are combinations of the off-axis fast and slow shear waves.

Our own analysis of this event using the wavefield-decomposed seismogram gives an even more convincing result than previously. We recover a lag time of $3.55 \pm 0.2 \mathrm{~s}$ and a fast direction of $22 \pm 2^{\circ}$. Rotated into the correct orientation, the traces show a clear split between similar shear waveforms; if the phase were truly not split (that is, no anisotropy), no such orientation would be possible.

This result shows more splitting than can be explained solely by VTI in the upper mantle. The a priori assumption of VTI media means that the methodology of Saul and Vinnik will be unable to detect any splitting with a fast direction that is neither horizontal nor vertical: even a small deviation will cause cross-contamination of the components.

Furthermore, their simple inspection of the waveforms is a qualitative method, relying on a subjective pick of the two phases, with no way of assessing errors. Our method produces a quantitative measurement of the error, allowing us to reduce our initial data set of 290 measurements to the 35 that are constrained sufficiently to draw robust inferences. We are left with a highquality data set that we consider to be compelling evidence of mid-mantle anisotropy. James Wookey ${ }^{\star}$, J.-Michael Kendall*, Guilhem Barruol $\dagger$

${ }^{*}$ School of Earth Sciences, University of Leeds, Leeds LS2 9JT, UK

e-mail:m.kendall@earth.leeds.ac.uk

$\dagger C N R S$, Université Montpellier, 34095 Montpellier, Cedex 05, France

\footnotetext{
Wookey, J., Kendall, J.-M. \& Barruol, G. Nature 415, $777-780$ (2002).

2. Silver, P. G. \& Chan, W. W. Nature 335, 34-39 (2002).

3. Clitheroe, G., Gudmundsson, O. \& Kennett, B. L. N. J. Geophys. Res. 105, 13697-13713 (1998).

4. Bostock, M. G. J. Geophys. Res. 103, 21183-21200 (1998).
} 